\title{
Automated Calculation of Infarct Transmurality
}

\author{
E Heiberg, H Engblom, M Ugander, H Arheden \\ Department of Clinical Physiology, Lund University, Sweden
}

\begin{abstract}
The aim of this study was to develop an algorithm to automatically calculate infarct transmurality based on a non dichotomous infarct classification, and to compare with manual delineation.

Global transmurality as calculated by the computer algorithm were significantly smaller than the consensus delineation of three observers $(p<0.05)$.

On a regional basis in 6 sectors of each slice the variability of the three observers compared to consensus delineation was $17 \%, 15 \%$, and $20 \%$. The variability of the automated algorithm was $16 \%$.

In conclusion, weighted calculation of transmurality gave smaller global transmurality compared to consensus delineation, but did had the same variability on a regional basis.
\end{abstract}

\section{Introduction}

Infarct size and transmurality are important determinants of prognosis after myocardial infarction [1]. Infarct size can be measured by using contrast delayed enhancement MRI (DE-MRI). There have been many approaches to automatically calculate infarcted myocardium from DE-MRI [2-8]. A common denominator of all these methods is that they all try to determine an image intensity threshold above which pixels are treated as completely hyperenhanced. Instead we have proposed an approach where pixels are not dichotomously classified as hyperenhanced or not [9]. In this approach each pixel that is classified as hyperenhanced after myocardial infarction is weighted with the pixel intensity to compensate for partial volume effects. Partial volume effects may cause one image pixel to be partially hyperenhanced or gray. Although partial volume effects have been suggested as a potential source of error in DE-MRI [10-12] to our knowledge no one has up until now incorporated a compensation for partial volume effects when designing automated methods for quantification of infarct size.

\section{Aim}

The aim of the study was to extend the previously proposed weighted algorithm to be able to calculate infarct transmurality, and compare these results with manual delineation.

\section{Methods}

For the previously developed automated infarct quantification algorithm [9], each pixel is assigned an infarct percentage. In order to assess infarct transmurality the following two steps are required.

\subsection{Region of hyperenhancement}

The first step in the process is manual delineation of both endocardium and epicardium. This process can also be made semi-automatically [13]. The algorithm applied to find the region of hyperenhancement is based on finding a threshold between hyperenhanced and normal viable myocardium based from number of standard deviations from remote. This is then combined with a 3D post processing method that restricts the hyperenhanced region to be spatial contiguous both in the in plane and through plane direction [14]. The number of used standard deviations from remote myocardium was optimized and calibrated by comparing the result of the algorithm on in vivo images with high resolution ex vivo images in 8 pigs as a reference standard [9].

\subsection{Calculation of infarct transmurality}

To calculate infarct transmurality the infarct percentage was integrated along radial spikes of the myocardium. The complete algorithm was implemented in the freely available software Segment (http://segment.heiberg.se). 
An illustration of the user interface is shown in Figure 1 below.

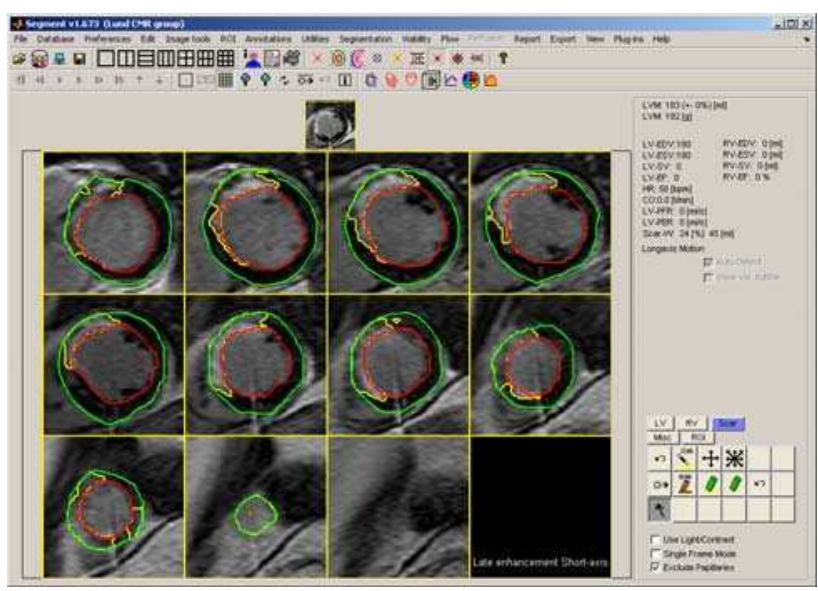

Figure 1. Screenshot of the freely available software.

Infarct transmurality was visualized in a bullsye plot according to the 17 segment model endorsed by AHA [15] (Figure 2).

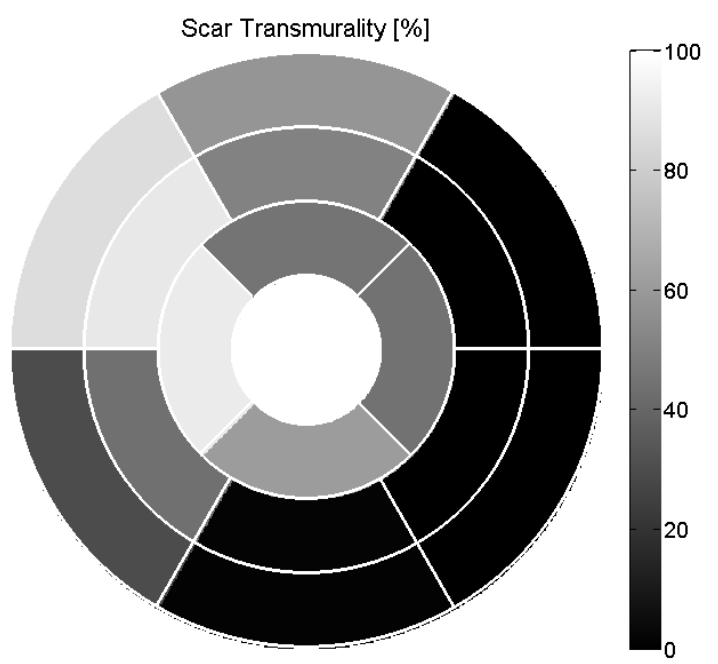

Figure 2. Example of segmental output from the software.

\subsection{Patient population}

The patient participants used in this study have previously been described [14]. In short, 20 patients had acute first time ST-elevation infarcts (all men, age $62 \pm 11$ years, age range 41-84 years, time between infarct and MRI was 8 \pm 1 days, range 6-10 days). Another group of 20 patients had been clinically referred for viability

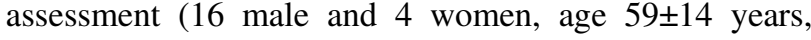
range 23-78 years). No patient was excluded based on poor image quality.

\subsection{Image acquisition}

For both patient groups (acute and chronic), half of the patients were scanned on a $1.5 \mathrm{~T}$ Siemens Vision Magnetom scanner (Siemens, Erlangen, Germany), and the other half on a 1.5T Philips scanner.

Imaging was performed in the short-axis plane during end-expiratory apnea. Image resolution was $1.6 * 1.6 * 8$ $\mathrm{mm}$, gap $2 \mathrm{~mm}$ (Siemens) or 1.56*1.56*8 mm, gap $0 \mathrm{~mm}$ (Philips). Imaging parameters were TR/TE: 3.8 $\mathrm{ms} / 1.1 \mathrm{~ms}$, flip angle $25^{\circ}$, FOV $400 \mathrm{~mm}$, matrix size $240 * 180$, inversion time typically 230-290ms (Philips), TR/TE: $250 \mathrm{~ms} / 3.4 \mathrm{~ms}$, flip angle $15^{\circ}$, FOV $410 \mathrm{~mm}$, matrix size $256 * 192$, inversion time typically $150-210 \mathrm{~ms}$ (Siemens).

\subsection{Comparing automated method to manual delineation}

Three observers blinded to each others results manually outlined infarct region in 40 patients. All three observers used the same delineation of the endo- and epicardium. A consensus delineation was defined as mean of the three observers. Infarct transmurality was quantified both globally and regionally for each patient. Global infarct transmurality was defined as the mean infarct transmurality of all myocardium that was indicated as hyperenhanced. Regional transmurality was quantified in 6 sectors in each MRI short axis slice.

\section{Results}

Results are presented as mean $\pm \mathrm{SD}$, and measured as difference between observers and the automated algorithm.

\subsection{Global results}

Mean global transmurality for the three observers, and computer algorithm were: $35 \%, 31 \%, 38 \%$, and $28 \%$, respectively. Differences compared to consensus delineation were (mean \pm SD): $-0.6 \pm 4.4 \%,-4.2 \pm 5.3 \%$, $3.6 \pm 5.5 \%$, and $-6.7 \pm 13 \%$, respectively. The three 
observers were not statistically different from each other (one-way ANOVA, $\mathrm{p}=0.18$ ). Transmurality as calculated by the computer algorithm were significantly smaller than the consensus delineation $(\mathrm{p}<0.05)$.

\subsection{Regional results}

For the three observers, and the computer algorithm the mean transmurality in infarcted sectors were: $44 \%$, $31 \%, 49 \%$, and $30 \%$, respectively. Number of infarcted sectors were: $962,1324,789$, and 1449 . The differences compared to consensus delineation were for the three observers $6.7 \%, 2 \%$, and $-9 \%$. The difference compared to consensus delineation for the computer algorithm was $3.2 \%$. The variabilities of the three observers compared to consensus delineation were $17 \%, 15 \%$, and $20 \%$, respectively. The variability of the computer algorithm compared to consensus delineation was $16 \%$.

The three observers differed significantly from each other (one-way ANOVA, p<0.01). The computer algorithm was significantly different from consensus $(\mathrm{p}<0.01)$.

\section{Discussion and conclusions}

Quantification of infarct transmurality is difficult since the left ventricular wall is relatively thin compared to the pixel resolution. As an example, in a normal ventricle the wall is about $12 \mathrm{~mm}$ thick, given a pixel resolution of 1.5 $\mathrm{mm}$ one single pixel difference will give a difference in transmurality of $13 \%$. Therefore using a weighted approach to calculate transmurality has the potential to reduce the variability since it works on a sub-pixel level. On a global level the automated algorithm showed lower infarct transmurality compared to manual delineation. On the regional scale the variability is substantial between the three observers, indicating that regional assessment of infarct transmurality is difficult unless the sectors are sufficiently large to average out the some of the variability.

Weighted calculation of infarct size shows smaller variability compared to dichotomous approaches [9]. Further studies are merited in order to determine if this also applies for weighted calculation of transmurality as proposed in this study.

In conclusion, weighted calculation of transmurality gave smaller global transmurality compared to consensus delineation, but did had the same variability on a regional basis as manual delineation.

\section{Acknowledgements}

The authors would like to express their appreciation to Dr. Jan Engvall, Linköping University hospital for being one of the three observers.

This work was supported in part by the Swedish Research Council, the Swedish Heart and Lung Foundation, the Faculty of Medicine at Lund University, and the Region of Scania.

Address for correspondence

Einar Heiberg

Department of Clinical Physiology

Lund University Hospital

22185 Lund, Sweden

Einar.Heiberg@med.lu.se

\section{References}

[1] Kim RJ, Wu E, Rafael A, Chen EL, Parker MA, Simonetti $\mathrm{O}$, et al. The use of contrast-enhanced magnetic resonance imaging to identify reversible myocardial dysfunction. $\mathrm{N}$ Engl J Med. 2000 Nov 16;343(20):1445-53.

[2] Setser RM, Bexell DG, O'Donnell TP, Stillman AE, Lieber ML, Schoenhagen $P$, et al. Quantitative assessment of myocardial scar in delayed enhancement magnetic resonance imaging. J Magn Reson Imaging. 2003 Oct;18(4):434-41.

[3] Amado LC, Gerber BL, Gupta SN, Rettmann DW, Szarf $\mathrm{G}$, Schock R, et al. Accurate and objective infarct sizing by contrast-enhanced magnetic resonance imaging in a canine myocardial infarction model. J Am Coll Cardiol. 2004 Dec 21;44(12):2383-9.

[4] Positano V, Pingitore A, Giorgetti A, Favilli B, Santarelli MF, Landini L, et al. A fast and effective method to assess myocardial necrosis by means of contrast magnetic resonance imaging. $\mathrm{J}$ Cardiovasc Magn Reson. 2005;7(2):487-94.

[5] Schuijf JD, Kaandorp TA, Lamb HJ, van der Geest RJ, Viergever EP, van der Wall EE, et al. Quantification of myocardial infarct size and transmurality by contrastenhanced magnetic resonance imaging in men. Am J Cardiol. 2004 Aug 1;94(3):284-8.

[6] Bondarenko O, Beek AM, Hofman MB, Kuhl HP, Twisk JW, van Dockum WG, et al. Standardizing the definition of hyperenhancement in the quantitative assessment of infarct size and myocardial viability using delayed contrast-enhanced CMR. J Cardiovasc Magn Reson. 2005;7(2):481-5.

[7] Heiberg E, Engblom H, Engvall J, Hedström E, Ugander M, Arheden H. Automated quantification of delayed contrast-enhanced MR images. SCMR; 2006; Miami; 2006. 
[8] Hsu LY, Natanzon A, Kellman P, Hirsch GA, Aletras AH, Arai AE. Quantitative myocardial infarction on delayed enhancement MRI. Part I: Animal validation of an automated feature analysis and combined thresholding infarct sizing algorithm. J Magn Reson Imaging. 2006 Mar;23(3):298-308.

[9] Heiberg E, Ugander M, Engblom H, Götberg M, Olivecrona G, Erlinge D, et al. Accurate and automated quantification of myocardial infarction from delayed enhancement MRI by accounting for partial volume effects. Radiology. In press.

[10] Goldman MR, Brady TJ, Pykett IL, Burt CT, Buonanno FS, Kistler JP, et al. Quantification of experimental myocardial infarction using nuclear magnetic resonance imaging and paramagnetic ion contrast enhancement in excised canine hearts. Circulation. 1982 Nov;66(5):1012-6.

[11] Kim RJ, Fieno DS, Parrish TB, Harris K, Chen EL, Simonetti O, et al. Relationship of MRI delayed contrast enhancement to irreversible injury, infarct age, and contractile function. Circulation. 1999 Nov 9;100(19):1992-2002.
[12] Judd RM, Lugo-Olivieri CH, Arai M, Kondo T, Croisille P, Lima JA, et al. Physiological basis of myocardial contrast enhancement in fast magnetic resonance images of 2-day-old reperfused canine infarcts. Circulation. 1995 Oct 1;92(7):1902-10.

[13] Heiberg E, Wigström L, Carlsson M, Bolger AF, Karlsson M. Time Resolved Three-dimensional Automated Segmentation of the Left Ventricle. IEEE Computers in Cardiology 2005; 2005; Lyon, France: IEEE; 2005. p. 599602.

[14] Heiberg E, Engblom H, Engvall J, Hedstrom E, Ugander $M$, Arheden $H$. Semi-automatic quantification of myocardial infarction from delayed contrast enhanced magnetic resonance imaging. Scand Cardiovasc J. 2005 Oct;39(5):267-75.

[15] Cerqueira MD, Weissman NJ, Dilsizian V, Jacobs AK, Kaul S, Laskey WK, et al. Standardized myocardial segmentation and nomenclature for tomographic imaging of the heart: a statement for healthcare professionals from the Cardiac Imaging Committee of the Council on Clinical Cardiology of the American Heart Association. Circulation. 2002 Jan 29;105(4):539-42. 